\title{
An Analytic Study on the Identification of the Stress Softening Region of the Compression-type Sleeve for CFRP Tendons
}

\author{
Woo-tai Jung ${ }^{1, a^{*}}$,Jae-yoon Kang ${ }^{2, b}$, andMoon-seoungKeum ${ }^{3, \mathrm{c}}$ \\ ${ }^{1}$ Structural Engineering Research Division, Korea Institute of Civil engineering and Building \\ Technology, (Daehwa-Dong)283, Goyangdae-Ro, Ilsanseo-Gu, Goyang-Si, Gyeonggi-Do, Korea \\ ${ }^{2}$ Structural Engineering Research Division, Korea Institute of Civil engineering and Building \\ Technology, (Daehwa-Dong)283, Goyangdae-Ro, Ilsanseo-Gu, Goyang-Si, Gyeonggi-Do, Korea \\ ${ }^{3}$ Structural Engineering Research Division, Korea Institute of Civil engineering and Building \\ Technology, (Daehwa-Dong)283, Goyangdae-Ro, Ilsanseo-Gu, Goyang-Si, Gyeonggi-Do, Korea \\ aemail:woody@kict.re.kr, bemail:jykang@kict.re.kr, ${ }^{\mathrm{c} e m a i l: m o o n s e o u n g @ k i c t . r e . k r ~}$
}

Keywords:Carbon Fiber Reinforced Polymer, Compression-type Anchor, Strengthening, Taper, Tendons

\begin{abstract}
Compared to the bond-type anchor, the compression-type anchor, whichanchors the CFRP tendon by applying a compressive force on its surface, presents the advantages of not needing a curing period for the filler and to reduce the sleeve length by more than $30 \%$. The fabrication of the compression-type anchor proceeds by changing the dimensions of the steel sleeve by hydraulic pressure, which means that the anchoring performance varies according to the region subjected to the compressive force at the surface of the CFRP tendon. Since the fabrication of the compression-type anchor is generally done uni-directionally, there is naturally some difference in the performance at the loaded end and free end. Accordingly, it is necessary to design the stress softening region to secure uniform anchoring performance at both ends. This study performs finite element analysis on 7 different shapes of sleeves with two types of taper based upon the dimensions of existing sleeves. From the analysis results, the design of the stress softening region of the free end is not possible by adjusting only the stress softening region but needs also to adjust the inner diameter of the sleeve so as to obtain similar stress softening regions in both loaded and free ends. For the sleeve with two tapers, similar stress softening regions could be achieved after compression by securing a stress softening region longer than $30 \mathrm{~mm}$ at the free end. This disposition was also seen to secure longer iso-stress region than the other parameters, from which it can be expected to acquire stable results during the test of the loaded and free ends.
\end{abstract}

\section{Introduction}

Steel and concrete are the most important construction materials to date. However, following the demand for improved performance and durable structures, research has shifted toward the development of replacement materials and higher performance for the existing materials. FRP (Fiber Reinforced Polymer) has been studied to replace steel and solve the corrosion problem. It is now exploited as high performance material for the repair and strengthening of structures. Because of its low material resistance against transverse shear, FRP has encountered various problems for anchoring when evaluating its material performance or when used in jacking devices. The current methods for anchoring FRP can be classified into the wedge, bond and compression-type anchors. The wedge-type anchor operates by gearing mechanically the surface of the tendon using wedges. It is usually used for prestressing wires or strands. The bond-type anchor makes use of filler like expansive agent or resin injected in a steel tube to secure its anchoring performance by bonding the tendon and the filler. Compared to the two other types of anchors, the bond-type anchor provides the most reliable anchoring performance with minimal risk of damage of the tendon but requires very long and large sleeve since its anchoring performance depends on the bonding performance, itself depending on the external shape of the tendon and its cover. 
The compression-type anchor anchors the tendon by applying a compressive force on its surface by compressing the outer of the steel sleeve. Compared to the bond-type anchor, the compression-type anchor presents the advantages of not needing a curing period for the filler and to reduce the sleeve length by more than $30 \%$. Jung et al. [3] conducted an experimental study on various variables including the dimensions of the sleeve and the eventual presence of insert to examine the anchoring performance of the compression-type anchor. These authors found out that the anchoring performance of the compression-type anchor depends on the change in the swaging pressure exerted according to the inner and outer diameters of the sleeve, and reported the need to avoid stress concentration at the end of the sleeve by disposing a stress softening region in order to prevent the rupture of the tendon at the end of the sleeve. They derived the optimal dimensions of the steel sleeve with regard to the static stress so as to secure the anchor performance of the tendon by finite element analysis of the development of stress according to the change in the inner diameter of the sleeve.

In the previous compression-type sleeve, a tapered section is disposed along a definite region delimited by the starting position of the swaging pressure so as to create a stress softening region in which the compressive force in the CFRP tendon increases gradually. Test was performed on the anchor by letting the stress softening part after compression be the loaded end and the part pushed during the compression be the free end. However, when static test is conducted by setting the free end as the part with the stress softening region and the loaded end as the pushed part, there is high risk of rupture of the CFRP tendon in the part without swaging due to the absence of stress softening region. Accordingly, this study intends to design and verify the performance of the sleeve in which there is a stress softening region in both loaded end and free end of the CFRP tendon after compression in a will to prevent such risk.

\section{Finite Element Analysis}

Summary of Analysis Model.The program ABAQUS v.6.5 is used for the parametric analysis. An axial symmetric model is adopted considering the symmetry of the steel tube. The dice is modelled as a rigid body. As shown in Fig. 1 andconsidering the actual fabrication process of the swaging sleeve, loading was applied through displacement control so as to push the rear side of the sleeve by $160 \mathrm{~mm}$ and let it pass through the dice to exert the swaging deformation and gear the CFRP tendon by friction. At that time, the steel sleeve and the CFRP tendon are subjected to contact boundary conditions. Under such conditions, the coefficient of friction between the sleeve and the tendon is set to a conservative value of 0.15 due to the absence of studies on the coefficient of friction between the CFRP tendon and the steel sleeve and with reference to the approximate value of 0.23 provided by Schön [4] for aluminum in contact with a carbon fiber epoxy composite. Hard contact is assumed in the direction vertical to the contact surface. For the behavior acting in the tensile direction after contact, boundary conditions enabling separation at the contact interface are applied. Note that frictionless condition is assumed between the dice and the sleeve. The material properties of the CFRP tendon and the steel sleeve are arranged in Table 1.

Table 1.Material properties of CFRP tendon and steel sleeve.

\begin{tabular}{|l|c|c|c|c|c|}
\hline Material & $\begin{array}{c}\text { Elastic } \\
\text { modulus } \\
{[\mathrm{MPa}]}\end{array}$ & $\begin{array}{c}\text { Yield } \\
\text { strength } \\
{[\mathrm{MPa}]}\end{array}$ & $\begin{array}{c}\text { Tensile } \\
\text { strength } \\
{[\mathrm{MPa}]}\end{array}$ & $\begin{array}{c}\text { Diameter } \\
{[\mathrm{mm}]}\end{array}$ & $\begin{array}{c}\text { Cross-section } \\
\text { al area } \\
{\left[\mathrm{mm}^{2}\right]}\end{array}$ \\
\hline CFRP tendon $(\phi 10.0)$ & 165,000 & - & 3,500 & 10.0 & 19.63 \\
\hline Steel sleeve & 200,000 & 400 & 569 & - & \\
\hline
\end{tabular}



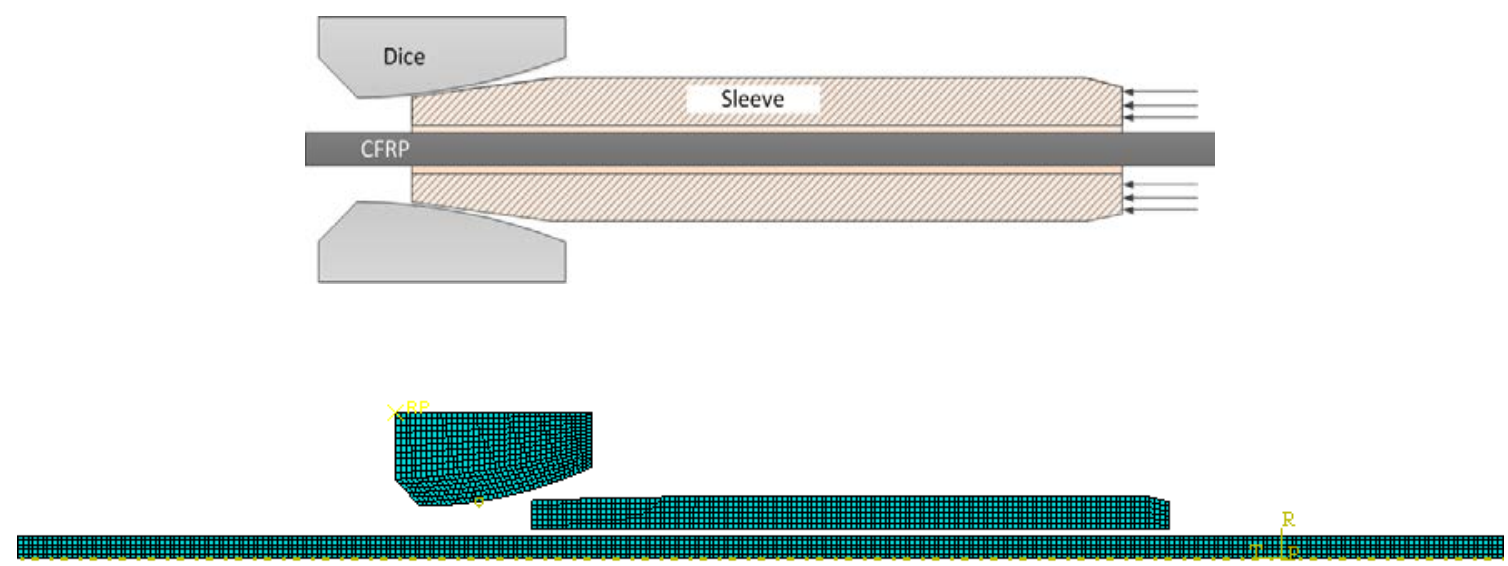

Fig. 1Loading method and analysis model.

Design of Stress Softening Region.Based upon the dimensions of the sleeve adopted by Jung [3] for a $\phi 9.5 \mathrm{~mm}$ CFRP tendon, finite element analysis is performed for the following basic shapes: the a-type, in which the stress softening region in the loaded and free ends is set as variable, and the b-type, which presents identical inner diameter to the a-type but with different length and diameter of the stress softening region at the loaded and free ends. Table 2 lists the characteristics of these different shapes. Here, the left-hand end of the sleeve is the loaded end and the right-hand end is the free end. The previous a-type sleeve presents a smooth taper in the stress softening region whereas the b-type exhibits two different tapers in order to compare the swaging pressures occurring at the surface of CFRP. Once the tensile performance is obtained in the $\phi 9.5 \mathrm{~mm}$ CFRP tendon, finite element analysis is performed in advance so as to generate a compression at the surface of the $\phi 10.0 \mathrm{~mm}$ CFRP tendon similar to the $350 \mathrm{MPa}$ developed at the surface of the $\phi 9.5 \mathrm{~mm}$ CFRP tendon and set the values of D1, D2 and D3. The shapes b2 and b3 present identical outer diameter and length but b2 has not the first taper at his ends while b3 is tapered.

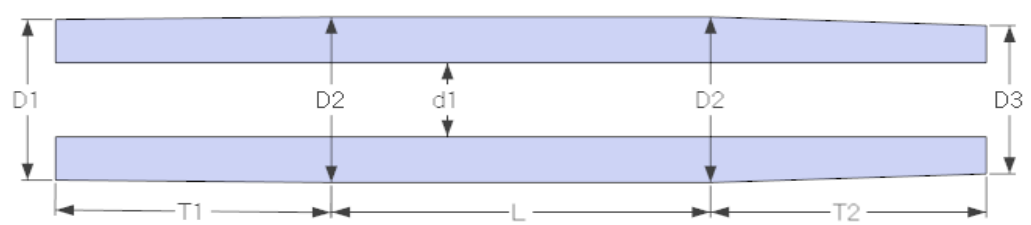

(a)

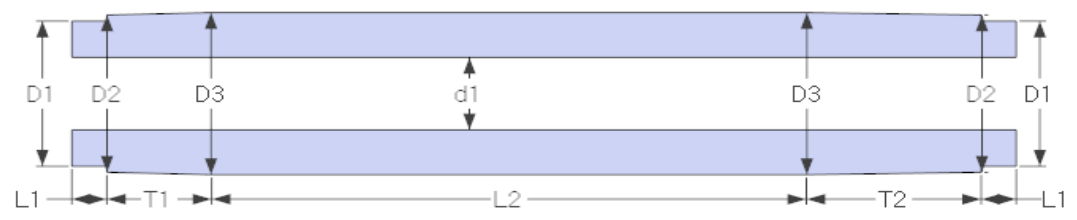

(b)

Fig. 2 Shapes of sleeves. 
Table 2.Dimensions of sleeves (in mm).

\begin{tabular}{|l|c|c|c|c|c|c|c|c|}
\hline Shape & D1 & D2 & D3 & d1 & T1 & T2 & L1 & L2 \\
\hline a1 & 26 & 26.8 & 24 & 12 & 40 & 15 & 80 & - \\
\hline a2 & 26 & 26.8 & 24 & 12 & 40 & 40 & 55 & - \\
\hline a3 & 26 & 26.8 & 26 & 12 & 40 & 40 & 55 & - \\
\hline a4 & 26 & 26.8 & 24 & 12 & 40 & 30 & 65 & \\
\hline b1 & 24 & 26 & 26.8 & 12 & 20 & 20 & 5 & 85 \\
\hline b2 & 24 & 26 & 26.8 & 12 & 20 & 30 & 5 & 75 \\
\hline b3 & 24 & 26 & 26.8 & 12 & 20 & 30 & 5 & 75 \\
\hline
\end{tabular}

Analysis Results.Fig. 3plots the change in the stress distribution occurring at the surface of the CFRP tendon all along the analysis process. It can be seen that a constant compression applies inside the sleeve after completion of the swaging. The compression along the effective swaging zone appears to be similar to that of the $\phi 9.5 \mathrm{~mm}$ CFRP tendon. Except for shape a3, the a-type shapes present a length of 15 to $40 \mathrm{~mm}$ for the stress softening region at the free end but without generation of a stress softening region as in the loaded end when applying a simple taper. This can be explained by the disappearance of the stress softening region when the sleeve is swagged from its left-hand side because the length of the sleeve increases and its diameter reduces while passing through the dice, which makes the diameter of the initially designed stress softening region increase due to the augmentation of the amount of steel at the free end. For shape a3, the length of the stress softening region seems to be $35 \mathrm{~mm}$ but this cannot be the case since the diameter $\mathrm{d} 3(=26 \mathrm{~mm})$ at the free end of the sleeve is larger than the inner diameter of the dice. At the exception of shape b1, the b-type shapes with two tapers show similar stress softening regions at the loaded and free ends. Accordingly, a stress softening region longer than $30 \mathrm{~mm}$ should be provided at the loaded end before swaging of the sleeve. Table 3 arranges the lengths of the stress softening region at the loaded and free ends as well as the length of the iso-stress region obtained analytically.

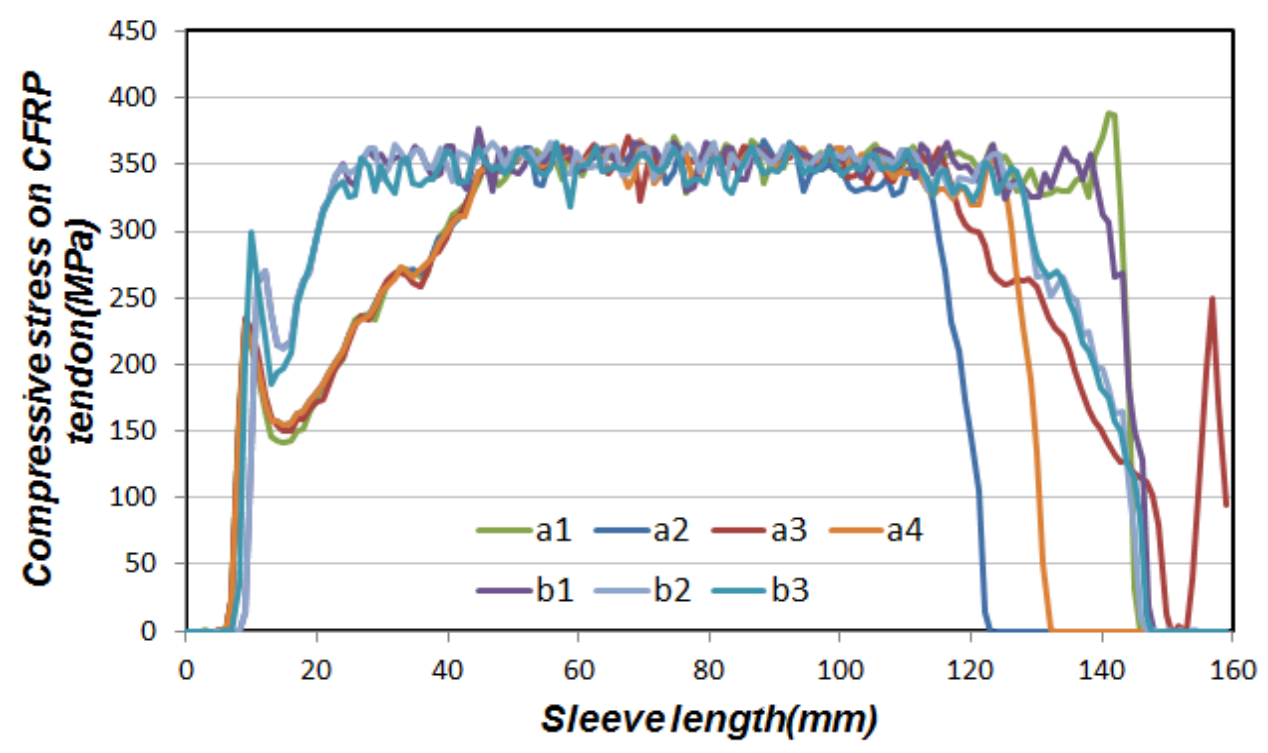

Fig. 3 Change in compressive stress in CFRP tendon according to dimensions of sleeve. 
Table 3.Summary of analytic results.

\begin{tabular}{|c|c|c|c|}
\hline \multirow{2}{*}{ Shape } & \multicolumn{2}{|c|}{ Stress softening region [mm] } & \multirow{2}{*}{ Iso-stress region [mm] } \\
\cline { 2 - 4 } & Loaded end & Free end & 95 \\
\hline a1 & 40 & 5 & 65 \\
\hline a2 & 40 & 10 & 65 \\
\hline a3 & 40 & 35 & 80 \\
\hline a4 & 40 & 10 & 110 \\
\hline b1 & 20 & 10 & 100 \\
\hline b2 & 20 & 20 & 100 \\
\hline b3 & 20 & 20 & \\
\hline
\end{tabular}

\section{Conclusions}

This paper presented an analytic study on the detailed design of the stress softening region at the free end of the compressive-type sleeve. The following conclusions could be derived.

Based upon the dimensions of existing sleeves, it appeared that not only the stress softening region but also the inner diameter of the sleeve should be adjusted for the design of the stress softening region at the free end.

For the b-type shapes with two types of taper, a stress softening region longer than $30 \mathrm{~mm}$ shall be managed at the free end in order to obtain a similar stress softening region at the loaded end after swaging. Since it appeared that the iso-stress region longer than the other variables should also be secured, it can be expected that stable results will be achieved during the test of the loaded and free ends.

\section{Acknowledgements}

This research was supported by a grant from a Strategic Research Project funded by the Korea Institute of Civil engineering and Building Technology.

\section{References}

[1] N.Nordin, B.Talisten, Concrete beams strengthened with prestressed near surface mounted CFRP, J. Compos. Constr. 10:1 (2006) 60-68.

[2] A. Hajihashemi, D.Mostofinejad, M.Azhari, Investigation of RC beams strengthened with prestressed NSM CFRP laminates,J. Compos. Constr. 15:6 (2011) 887-895.

[3] W.T. Jung, Y.H. Park, J.S. Park, An experimental study on improving anchor performance for CFRP tendons, in: FRPRCS-10, SP275-41, 275 (2011) 1-16.

[4] J. Schön, Coefficient of friction for aluminum in contact with a carbon fiber epoxy composite, Tribol. Int. 37 (2004) 395-404. 